\title{
6
}

\section{Towards A Methodology for 24 Hour Software Production Using Globally Separated Development Teams}

\author{
Ian Gorton and Sanjeev Motwani ${ }^{\mathrm{b}}$ \\ alan Gorton, School of Computer Science and Engineering, University of New South Wales, \\ PO Box 1, Kensington, Sydney 2033, Australia \\ ${ }^{\mathrm{b}}$ Sanjeev Motwani, Asia Pacific Systems Engineering Centre, BT Australasia, 1 Market St, Sydney \\ NSW 2000, Australia, E-mail: \{sanjeev, ian\}@apsec.bt.com.au
}

\begin{abstract}
The potential advantages of utilising software development teams which reside in different time zones are discussed. The experiences of an experimental study which attempted to share a software project between teams in Australia and India are then related. The results of this study show that, co-operative software development on a global scale is feasible, productivity gains are achievable and potentially enhanced product quality is an important by-product. ${ }^{+}$
\end{abstract}

Keyword Codes: K.6.1; K.6.3; D.2

Keywords: Project and People Management ; Software Management; Software Engineering

\section{INTRODUCTION}

As the global market for software continues its rapid growth, many multinational companies involved in software production are establishing development groups around the world. Ostensibly these groups usually aim to support the local customer base for their company's products, and on occasions may participate in strategic product development efforts. More often than not however, the development of a specific product is localised to a single site. This is in main due to the perception that developing complex software systems is an inherently difficult task, and introducing unnecessary communications barriers between the personnel involved will only make the task more difficult[1].

In this paper we argue that geographically separated development teams should be viewed as an opportunity to improve and re-focus an organisation's software development practices. With modem communications networks, support tools and suitable software engineering and management practices, significant time differences between teams can be exploited to reduce product development times.

We take the approach that a software development project may be divided amongst geographically separated teams possessing partially overlapping or disjoint working hours. These teams will have a fast and reliable data communications network, and may also communicate by telephone and facsimile. In order to make the time differences work to their advantage, the development teams must collaborate on a shared task. For example, one team will work upon the task during their normal working hours. At some stage before the end of their day part or all of the task will be handed over to a collaborating team to continue with during their normal working hours. Near or at the end of the collaborating team's working

\footnotetext{
${ }^{+}$Ian Gorton would like to thank BT Australasia for their support in the BT Research Fellowship scheme. Ian Gorton and Sanjeev Motwani would like to thank Mark Norris, David Beale, Martin Deacon, Udaykumar Revankar, Sunil Chavan, Michael Rohanek and Ponampalam Balakumar for their participation in this project.
} 
day, they will hand the task back for continuation by the original team. In this manner, a team would be able to build upon the progress made by the co-operating team 'ovemight' - a phenomena we call the overnight gain effect.

It is envisaged that the effective use of development teams across time-zones could have a number of beneficial effects for an organisation involved in software development Overlap of development activity, giving potentially 24 hour continuous development effort could significantly reduce time-to-market for software products. It could also lead to better utilisation of available skilled employees, minimising duplication of expert skills in different locations around the world. Product quality may further be enhanced due to the introduction of global perspectives during the development effort, and costs may be reduced by exploit low operating overheads and skilled staff in developing nations[2]

However, to exploit such a working environment, it will be necessary to implement procedures and methods to organise, monitor, control and synchronise distributed team efforts. For example, individual teams must be able to ascertain precisely which parts of a project have been completed, which are still in progress, and which are still to be tackled. Co-ordination of team activities will inevitably incur some overhead, and management of such a decentralised effort will be inherently more complex.

The motivation for this work arises from the establishment of a new BT software development centre in Sydney, Australia. For the Sydney group to actively and effectively participate in BTs global development processes, there is a need to extend and re-design existing development methodologies to take into account the problems and opportunities associated with distributed teams. Consequently, in order to understand the fundamental work factors which affect developers working in a globally distributed environment, we are conducting a series of progressively more complex experimental studies on live software projects. These will culminate in a large development trial using several teams in a number of locations. This paper describes the second study, which used two one-person teams located in Sydney and Bombay to develop a database package.

\section{MODELS OF DISTRIBUTED WORKING IN SOFTWARE DEVELOPMENT}

We propose three major models for organising distributed teams to take advantage of time differences: co-operative (peer-to-peer), delegation (supervisor/worker) and consultative (expert consultation). Each model has its own strengths and weaknesses, and can be used as a guide to match team organisations to project requirements and specific tasks.

When teams are organised to operate co-operatively, teams will exist on an equal basis and share closely related activities. For example, two teams may work on functionally dependent modules in a system. When the first team completes coding a module, it may pass the module and test specifications to the second team for testing. The second team will run the tests, and fix any errors which it can detect in a reasonable amount of time, only passing back problems to the first team in severe cases. In return, as the second team completes modules, it passes the code and test specifications to the first team for testing and repair where possible. Co-operating teams could also utilise each other for quality assurance activities. To effectively co-operate in such activities, teams should be comfortable when working together, be of similar status and possess a similar skill set. Effort in identifying tasks which are of suitable duration and complexity would also be required, and communication and management overheads would be unavoidable.

The delegation model of co-operation across time zones would seem most applicable to development, testing and support phases of software development. For example, a dedicated test team could accept completed modules and test specifications from a supervising development team. The test team would run tests and report back on their success and failure. The development team would then fix errors and pass the fixed code back for regression testing. A similar team structure could be operated during the maintenance and support cycle of a system. Supervisor/worker teams are not uncommon in large software developments [3]. Their advantage is a clear separation of responsibility between teams, easing control and management overheads. When operating in disjoint time zones, the scope for exploiting the 
overnight gain effect is significant, and reduced product development cycles could be expected. Further, the worker team does not require the experience or expertise of the supervisory team. This should lower the level of technical discussion between teams, giving concise and structured interactions, and reducing the likelihood of technical disputes.

Consultative teams would seem to offer broad scope for exploitation of the ovemight gain effect in software development. Expert teams with skills ranging from reviewing development proposals to enhancing existing systems could be deployed. In certain circumstances, these teams would be used as organisation-wide resources, in others they may be dedicated to a particular project. Tasks of short duration could be dealt with rapidly and feedback be returned to the client on their next working day.

\section{AN EXPERIMENT IN SOFTWARE DEVELOPMENT ACROSS TIME ZONES}

During September 1993 an initial experimental study was held within BT's Asia Pacific Systems Engineering Centre (APSEC) in Sydney. This study simulated two geographically separated single person teams by placing an imaginary barrier between two developers in the same office. This initial study was mainly used to test out how task assignments and synchronisation could be organised, and how each team's progress could be monitored and controlled.

With the experience of the first study, a more ambitious second experiment was planned. Two single person teams were established to operate using the delegation model to develop a PC-based database package. The program development team was based in Sydney, with the program test team based in Bombay, India. In addition a local project leader was assigned to each team to monitor overall progress and deal with organisational issues. The time difference between Sydney and Bombay is five and a half hours, thus allowing a three hour overlap of core working hours. This was deemed important as it would allow unforeseen problems to be immediately discussed and corrected over a telephone. The study was planned to operate for approximately one week during December 1993 with the aim of developing and testing the major input and query screens in the database project.

The Sydney team was given sole responsibility for designing and developing the software, and producing test specifications. A design specification for the project was completed prior to the study commencing: this acted as the base reference document for both teams. As parts of the system were implemented, they were passed over to the test team in Bombay, which ran the appropriate tests and reported back on their success or failure. Due to the time difference between the two teams, from the development team's perspective, testing would have occurred 'overnight', allowing the overall project to progress at a faster rate (Sydney is five and a half hours ahead of Bombay).

No direct data link existed between the Sydney and Bombay sites. A BT private network with reasonably fast delivery for small files was however available for email messages. File transfers between sites were thus achieved using a modem connection. While this was certainly slow compared with other forms of communication, the amount of data to be transferred daily was relatively small (maximum of $300 \mathrm{~KB}$ ) and consequently did not consume too much time.

For this mode of working to be successful, a number of project control and communications mechanisms were established to help overcome the distributed nature of the project teams.

\subsection{Task Assignment}

A Task Assignment (TA) form was designed to enable the development team to assign tasks to the test team. A TA was to be submitted by the development team each day, and it was to specify which modules should be tested and which test specifications should be used. In addition, any previously reported faults that had been fixed and should be re-tested were to be specified. As well as fields for general reference (e.g. source, destination, expected duration) the TA contained an area for allocating contingency work. Due to the nature of distributed work across time zones, unexpected problems (e.g. corrupt files, ambiguity in test specifications) could delay a project for longer than when such problems can be 
discussed face to face. In such circumstances, the contingency work allocation would be used to guide the test team to work on areas that could be profitably exploited in the near future.

\subsection{Test Specifications and Fault Reports}

Test specifications were drawn up by the development team. For each user screen in the system, a detailed list of tests were specified. A set of standard test cases was also created to ensure overall consistency between screens in the system. Whenever a test case in a test specification produced an error, the test team was to create a Fault Report (FR). A FR must contain sufficient information to allow the error to be reproduced by the development team. Each FR submitted by the test team was to be listed in the daily status report to ensure that FR's did not for some reason 'go missing', and to provide a check-list for the development team.

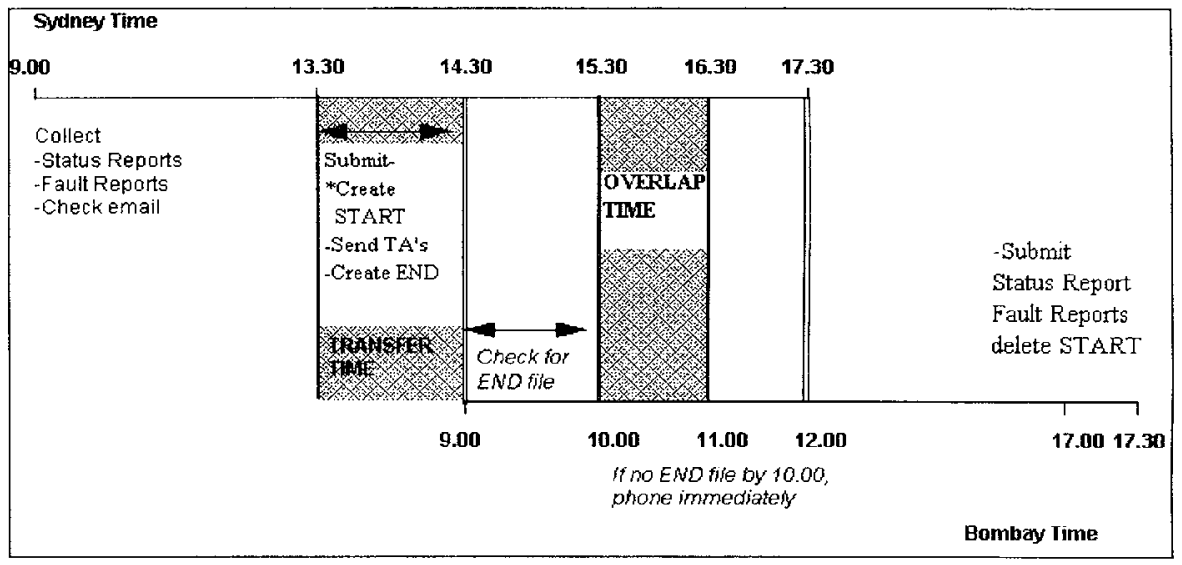

\subsection{Status Reporting}

Figure $1 \quad$ Daily Schedule for Development and Test Teams

A status report (SR) was to be submitted by the test team using email at the end of each day. The SR should indicate exactly what has been accomplished and what problems have occurred. This information should include the exact status of each task that has been assigned, and identify tests that have been completed and test cases which will require action by the development team.

\subsection{File Transfers}

Two distinct types of file transfers are required in a distributed team project. First, the current files associated with the project development such as program code, executables, test data and test specifications must be maintained in a common area. When using the delegation model, only the development team is allowed to alter these files. Consequently the development team must ensure that the latest versions of all development-related files are made available to the test team. In general these files are too numerous and large to be conveniently or reliably transferred by electronic mail. Second, project control files such as TA's, SR's and FR's must be exchanged daily. TA's are transferred from the development team to the test team, and SR's and FR's are transferred from the test team to the development team. In general these files are small in size (one or two pages of text) and can be transferred conveniently via electronic mail.

It is important to note that the successful transfer of both categories of files is not alone a sufficient condition for progress. Not only must the files be transferred successfully, they must also be available at the start of the working day of the team which is to utilise the files. Consequently, a daily schedule of activity was drawn up to clearly specify the deadlines by which certain activities were to take place. This 
daily schedule is shown in Figure 1. Two important time periods are highlighted in the schedule, namely transfer time and overlap time. Transfer time is the period allocated for the development team to make the latest versions of the project files available to the test team. To ensure partial transferred files were not accessed during transfer time, a simple file locking mechanism (using the START and END files in Figure 1) was implemented. Overlap time was the period designated for direct telephone conversations between the two teams.

\section{EXPERIMENTAL OBSERVATIONS}

The study ran from the 15th to 22 nd December. For the majority of this time, program development and testing successfully overlapped. Each day newly developed screens were passed to the test team during transfer time. The development and test team then proceeded independently until the end of the development team's work day. The test team continued testing until their work day ended, and transferred back status and fault reports for the development team to collect when they returned in the morning. In this manner, enhanced productivity in terms of actual progress on the project during the trial period was achieved due to the overnight gain effect. Many lessons were leamed in the process of designing, performing and monitoring the experimental study. Some of these are summarised below.

High Quality Planning is Essential: We consider the planning stage to be crucial to a project's success. The scope for severe problems to arise when using distributed teams is considerable. It must therefore be absolutely clear what the roles and responsibilities of each team are, and how they are to communicate and interact[1]. Soliciting input from the development teams during the planning stage, and obtaining feedback on plans ensures that team members are well informed and leads to project guidelines which everyone is content to follow.

Ensure that communications networks are adequate: A reliable and fast communications network is essential for projects which use globally separated teams, as failures in the network have the potential to cause havoc. Projects should ideally utilise existing networks which have been in use for a reasonable period of time and for which the performance characteristics and failure rate are well known. Well before a project commences, estimates of the communications load should be made. This should involve estimates of the maximum file size that will need to be transferred, the amount of files transferred on a daily basis, and overall traffic expected on the network. In addition, a back-up network should be established to ensure that the project can continue co-operatively in the event of a network failure

Monitor File Transfers: It should never be assumed that file transfers are successful. Measures must be instigated to monitor all file transfers and ensure that some form of crash recovery mechanism is automatically invoked.

Review All Project Documentation: When direct contact amongst team members is not possible, minor inconsistencies, mistakes or ambiguities in project documentation can potentially cause a delay of a day or more due to time differences. To help remove these problems, quality assurance methods such as peer review should be utilised extensively on all materials which will be passed to other sites.

Allocate Multiple Tasks: Despite all attempts to eradicate errors and provide reliable communications, problems inevitably occur. It is therefore essential that the problem does not stop a team from performing any productive work until the issue can be cleared up - this could mean a whole day of lost productivity. To guard against such delays, multiple, independent tasks should be allocated and be current at any particular time. This allows a team to continue working on other parts of the project until obstacles on a task are removed. In our trial, contingency tasks in TA's played this role effectively.

Minimise Distractions to Key Team Members: If a strong daily dependence exists for work allocations between two teams, distractions which delay one team temporarily can adversely affect the related team. This kind of problem can be minimised by ensuring that sufficient tasks are outstanding so that temporary productivity lapses have little overall impact (see previous point).

Problem Resolution: A project needs problem resolution procedures for technical or managerial issues which arise as the project progresses. This can be especially problematic in projects with 
distributed teams, as the potential for direct communications is limited. An important issue for one team may not seem so crucial from another team's perspective. Other problems may call for some synchronised action at each site. If one team neglects to perform this action, all other teams are delayed. In our study we defined a shared issues table to track and maintain the status of all unresolved problems. Each team leader was assigned the responsibility of maintaining this table, and ensuring that appropriate actions were taken. However, despite taking these measures, not all problems were dealt with in an effective and timely manner. For example, some requests in email or fax messages were missed or ignored, and occasionally incorrect actions were carried out due to a lack of background information. It was felt that the distributed responsibility for problem resolution (amongst team leaders) was not effective. One possible solution would be to assign a single person to be responsible for global problem resolution. In this manner, a project-wide perspective on problems could be maintained.

Be Prepared for Cultural Differences: Considerable differences in cultural backgrounds and attitudes towards work are seen amongst western societies. These differences are even greater when teams from diverse, non-western nations participate in a project. Some examples of the cultural differences encountered in our trial were varying degree of command over written and spoken English, differing work ethic, and the expected level of formality in communications.

Choose Key Team Members Carefully: Working in a distributed environment places additional pressure and demands on key team members. We observed that communications failures between sites cause genuine emotional distress, and the inherent reliance on other teams for progress causes pressure to mount to considerable levels. For these reasons, experienced and highly motivated staff should be used to fill important positions. Moreover, good communications and negotiation skills are essential.

\section{FURTHER WORK AND CONCLUSIONS}

The potential advantages of deploying globally separated software development teams have been presented, and three models for their organisation have been discussed. In performing an experiment in distributed working across time zones, some important issues and potential problems were observed, and guidelines for running such a project were tested. At this stage, after two small-scale studies in distributed working, considerable insight into the inherent problems of this approach has been developed. Several solutions have been proposed and validated during both studies. Next we intend to further test these solutions in a more extensive study involving more personnel and a larger time difference between teams. While the project has so far focused on exploiting a delegation development model, we aim to experiment with the co-operative model. Although the problems of teams working co-operatively are probably the most significant, we feel this may have the greatest potential for exploiting concurrent activities using globally separated teams[4].

The project will also consider how $\mathrm{CSCW}$ tools could help distributed teams interact and share information. We envisage that work flow and scheduling system in the manner of that proposed in [5] could help considerably with activity co-ordination. Such a system would provide a unified view of the project development files, and control access and file transfers between sites.

\section{REFERENCES}

1. Truett,L.F. et al, Co-ordination of Software Development Activities Among Sites that are Geographically Separated, in COMPSAC-17, (Nov 1-5, Phoenix), IEEE, 1993, pp 70-75

2. Press, L., Software Export From Developing Nations, IEEE Computer, 26, 12, (1993), pp 62-67

3. Norris, M and Rigby, P., Software Engineering Explained, John Wiley and Sons, UK, 1992

4. Prasad, B., Morenc, R.S. and Rangan,R.M., Information Management for Concurrent Engineering: Research Issues, Concurrent Engineering: Research and Applications, 1, 1, March 1993, pp 3-20

5. Gronbaek, K., Kyng, M., and Mogensen, P., CSCW Challenges: Co-operative Design in Engineering Projects, Communications of the ACM, 36, 4, (June 1993), pp 67-77 